\title{
Contribution à l'histoire des établissements hospitaliers de Nyon
}

\author{
Par ROger Joris
}

La ville de Nyon possédait depuis le Moyen-Age deux établissements hospitaliers; la léproserie ou maladière d'une part et un hôpital d'autre part.

La date exacte de fondation ne nous est connue ni pour l'une, ni pour l'autre de ces deux maisons. Mais certains documents nous permettent tout de même de situer avec une approximation convenable cette date.

Commençons tout d'abord par la léproserie. Elle était située à l'extrémité du territoire actuel de la commune en direction de Genève, en un lieu appelé Colovrex. Actuellement on y trouve une gravière et une place de camping.

Les documents concernant cette maladière sont peu nombreux au début, et même presqu'inexistants en ce qui concerne son premier siècle d'activité. Des recherches ultérieures permettront peut-être de découvrir des manuscrits inconnus pour l'instant.

Il n'y a que fort peu de chose à ajouter au remarquable travail fait par le Dr E. Olivier, dont nous ne pourrons jamais assez regretter la disparition. Ce travail parut en 1943 dans la Revue Historique vaudoise; et après 16 ans il n'y a, pour ainsi dire, rien à retrancher; comme j'ai pu mettre la main sur quelques documents qu'Olivier n'avait probablement pas pu consulter, cela me permet d'apporter ainsi une modeste contribution à son travail et à ses recherches sur la maladière. Par contre je n'ai trouvé aucun texte d'Olivier traitant de l'hôpital et de son histoire.

Selon le Dictionnaire historique vaudois de Motтaz, l'établissement daterait de 1244. Selon Olivier, il serait encore antérieur à cette date. Aucun des documents que j'ai consultés ne me permet de vérifier cette date, mais aucun non plus ne me permet de l'infirmer. Je crois pouvoir dire toutefois que si cette date n'est pas absolument exacte, l'erreur ne doit pas être considérable, si l'on s'en tient à un document datant de 1321 et qui permet de tirer quelques conclusions.

Voici ce qu'il en est.

Le 29 janvier 1321 (quarto Kalend. januar. 1322), par devant Perret (ou Pernet) de Divonne, clerc et notaire, et Jaquet, fils de Janin d'Aubonne, vidomme de Divonne, les habitants de la maladière déclarent que leur 
établissement a été fondé par Messire Guerry d'Aubonne, chevalier. Or ce Guerry est le grand-père de Jaquet présent au moment de l'acte.

Malgré cette affirmation, il reste pourtant un doute, tant sur la date, que sur son fondateur.

En 1493, lors de la nomination d'un recteur pour la maladière, il est bien spécifié que la ville de Nyon a droit de nomination du recteur de la léproserie «parce que cette léproserie lui appartenait, comme par elle fondée et établie dans son propre territoire». D'après l'acte de 1321 , nous pouvons connaître la situation assez exacte du bâtiment principal et de ses dépendances; le four, la chapelle, l'habitation du recteur, père spirituel de la communauté des malades.

On constate que le nombre des lépreux hospitalisés n'a jamais été considérable; Olivier en compte une quarantaine en deux siècles et s'étonne de ce nombre restreint. Je ne pense pas qu'il y ait lieu de s'étonner de la chose, car la lèpre était en voie de disparition au début du $\mathrm{XVI}^{\mathrm{e}}$ siècle dans notre pays. Il restait bien quelques lépreux, mais leur nombre allait en diminuant régulièrement. A Genève, à la même époque, il y avait encore deux léproseries dont les pensionnaires étaient fort peu nombreux pour un territoire bien plus vaste que celui de Nyon. Vers 1537, la léproserie de Carouge n'abritait déjà plus que deux ou trois ladres. Aucun document ne nous permet de penser que la région nyonnaise fût particulièrement éprouvée par cette terrible maladie.

Dès la fin du XVI $\mathrm{XV}^{\mathrm{e}}$ siècle, la lèpre ne faisait pratiquement plus de nouvelles victimes dans nos régions. Il y avait certainement encore des lépreux qui achevaient leur misérable existence dans les différentes maladières du pays.

Mais la maison de Nyon ne disparut pas pour autant; elle avait d'ailleurs été reconstruite en 1551, à la suite d'un incendie probablement, ou tout simplement parce qu'elle tombait en ruines. Quoi qu'il en soit, son utilité devait être encore certaine, puisque la ville de Nyon se décida à la reconstruire en faisant appel, pour cela, à la contribution des communes du ressort de notre ville.

Sur les terrains de la maladière, on trouvait aussi le gibet et les roues de la Justice de Nyon. Et parmi les obligations du recteur de la maladière, il y avait entre autres celle d'ensevelir les suppliciés.

Ces sinistres installations sont mentionnées dans un acte notarié du 24 mars 1451, à propos de la vente d'une vigne près du gibet. Cet acte est signé d'Egrège Etienne Seyrat, notaire à Nyon. 
Ce ne sont donc pas les bernois qui construisirent les potences et les instruments de torture situés à Colovrex, comme on l'a cru tout d'abord.

Lorsque la lèpre disparut, notre pays eut aussi à faire face aux effroyables épidémies de peste qui ravagèrent toute l'Europe. A Nyon on réclamait la bullette de santé aux étrangers qui pénétraient dans la ville; la police surveillait les établissements publics et les maisons privées pour se garder de la contagion. Mais la peste atteignit quand même la ville à plusieurs reprises. Je pense, mais c'est une hypothèse que je formule, que la maladière servit alors à héberger ces malades, car il était assez habituel de ne pas soigner les pestiférés en ville, mais au contraire de les éloigner et, au besoin, de leur construire des capites pour les isoler. La maladière reconstruite était un endroit tout trouvé. Mais encore une fois, je le répète, il s'agit d'une hypothèse, qu'il sera peut-être possible une fois de vérifier.

En 1676 la léproserie existait toujours. En effet, le texte d'un traité passé entre LL.EE. de Berne et la ville de Nyon stipule que «lesdits nobles et bourgeois de Nyon cèderont et remettront à LL. EE. en faveur de leurdit Château de Nyon tous les fiefs et censes directes qu'ils peuvent avoir et posséder au nom de leur dite ville et hôpital...

En récompense de quoi LL. EE. promettent et concèdent d'affranchir ladite ville et hôpital de toutes les censes directes... notament de celles dûes pour la maladerie...

Ce traité date du 9 décembre 1676.

Il fut ratifié par LL. EE. du Conseil de la ville de Berne, le 10 juillet 1685. Dans la copie du traité que j'ai consulté, il n'est pas fait mention expressément de la maladière dans la ratification.

Passé cette date, on ne trouve plus d'indication de la maladière, sauf pour en parler comme d'une chose ancienne ou pour situer topographiquement des terrains. Par contre, on trouve encore les fourches ou gibet, à la fin du XVIII ${ }^{\mathrm{e}}$ siècle.

On en trouve encore des représentations aux archives de Nyon et aux archives de Berne.

\section{L'hôpital de Nyon}

Nous ne sommes malheureusement pas mieux loti que précédemment pour trouver la date de fondation de l'hôpital. Nous savons qu'il existait déjà en 1356 car la ville de Nyon achète, en faveur de l'hôpital, une maison, le 26 mai 1356. Elle en achète une autre le 21 janvier 1357. Et ce n'est pas 
pour faire de ces maisons un hôpital, car le 8 mai 1363 la ville loue cette maison à un certain Johannot de Pisis, bourgeois de Nyon.

Nous constatons que l'hôpital lui-même fait d'assez nombreuses transactions immobilières; achat et vente de vignes ou d'autres terrains; d'autre part, il est l'heureux bénéficiaire d'un certain nombre d'héritages et de legs; il s'enrichit ainsi progressivement tout au long des années. Les revenus augmentent considérablement car l'hôpital loue ses terrains et ses maisons, soit en ville, soit au Bourg de Rive, soit dans la campagne environnante, même jusqu'à Gland et plus loin encore, puisque le 12 janvier 1488, Martin de Golion, bourgeois de Nyon, fait don à l'hôpital de la cense annuelle et redimable de 6 florins d'or et 9 sols dûe sur une maison située à Aubonne, dans le Bourg intérieur en la rue du Marché. Et c'est pour la fondation d'une messe à célébrer tous les lundis à perpétuité, par le chapelain de l'hôpital, dans la chapelle de l'hôpital ou dans celle de Saint-Jean hors les murs de la ville.

Mais dans ces ventes et achats, tout ne se passe pas toujours facilement; quelques fois l'âpreté au gain des uns ou des autres entraîne quelques complications. C'est ainsi qu'en 1492, le 11 mai, Vénérable Pierre Reverdil, Recteur de l'Hôpital, est obligé d'indemniser les filles de la veuve Pierre Gautier, car elles ont été lésées de la moitié du juste prix, lors de la vente d'une vigne et d'un pré, faite par leur mère au chapelain de l'hôpital. Cet honorable ecclésiastique semble avoir pris beaucoup de soin pour amasser une belle fortune pour lui et les siens.

Cette même année, c'est le commissaire ducal qui est en conflit avec le même recteur à propos d'une grange dont la propriété est contestée par les deux parties. En définitive, le 19 février 1490, le Juge des Fiefs ducaux, tant nobles que ruraux, dans la Châtellenie et Ressort de Nyon rend une sentence qui est, cette fois, favorable à l'hôpital.

Malgré quelques affaires brillantes, la gestion des biens de l'hôpital laisse à désirer et le rapport diminue, tant et si bien qu'il n'est plus que de dix florins en 1498. Nous verrons comment le trop ingénieux recteur de l'époque, c'est toujours Pierre Reverdil, va trouver le moyen d'augmenter les revenus fléchissants de son établissement.

En 1514, le 18 octobre, je ne sais ce qu'est devenu Pierre Reverdil, mais s'il est encore en vie, il n'est plus recteur de l'hôpital et son successeur, moins acharné à arrondir sa fortune personnelle, fait don, au contraire de tous ses biens à la ville pour son hôpital; ce chapelain est Vénérable Urbain Mottioux; il fait ce don au moment où il se démet de ses fonctions de recteur. 
En 1568 la ville de Nyon supplie LL.EE. de Berne de bien vouloir admodier à l'hôpital la dixme de Fallon et celle de la cure de Coinsins, car les revenus de l'hôpital sont insuffisants.

LL.EE. acceptent cette supplication et y ajoutent même une promesse, qui ne leur coûte guère, celle de donner à l'hôpital tous les biens du très vieux curé de Nyon, quand celui-ci mourra; cela ne peut tarder encore longtemps semblent dire LL.EE.!

En date du 20 mars 1573, les autorités bernoises accordent donc à l'hôpital « tout le dixme, tant bled, que vin et autres choses » et ceci pour trois ans. Et le 24 janvier 1604, on retrouve une prolongation de cette dîme pour dix ans.

En 1593, le 25 février, nous trouvons le texte d'un albergement passé par la ville de Nyon à Honorable François Bellon, bourgeois de Nyon, d'une maison située auprès de la porte de l'horloge et dans laquelle l'hôpital se trouvait auparavant.

Nous connaissons donc la situation à peu près exacte de l'hôpital jusqu'à la fin du $\mathrm{XVI}^{\mathrm{e}}$ siècle, et nous savons aussi qu'une petite chapelle $\mathrm{y}$ était attenante.

A partir de ce moment, l'hôpital est déplacé dans le bas de la ville, au Bourg de Rive, probablement dans l'ancien couvent des Frères Mineurs, qui avait été desaffecté au moment de la Réforme.

Le 10 juillet 1732, Noble Elie Frédéric de La Fléchère fait don à l'hôpital d'une maison, grange, jardins et appartenances situées au Bourg de Rive, proche de l'hôpital.

Mais en 1755, l'état des bâtiments est si lamentable qu'on décide de les détruire et de reconstruire un autre hôpital, ce qui fut fait.

Et c'est ainsi que le 12 janvier 1761 le nouvel hôpital est reconstruit. Mais le règlement de l'hospitalier restera le même qu'auparavant: "On ne changera point notre ancienne économie en assistant, comme par le passé, les personnes en cas de l'être.»

Il faut se souvenir en effet que cet hôpital, comme d'ailleurs tous les autres à cette époque, n'est pas destiné à soigner des malades, mais bien plutôt à hospitaliser les nécessiteux, les vieillards et les pauvres passants, les éternels pélerins ou réfugiés. A propos de ces hospices on peut dire ici ce que l'on en dit dans l'histoire générale de la médecine éditée chez Albin Michel. "A côté du type que l'on peut appeler urbain, il importe de mentionner le type rural, les innombrables «hospitia » du Moyen-Age, que l'on rencontrait le long des routes les plus fréquentées et qui étaient avant tout des asiles 
pour les voyageurs et les pélerins; cependant, on y recevait aussi les malades de la contrée... »

Il fallut attendre encore plus d'un siècle avant que l'hôpital fut transformé en infirmerie.

C'est en effet le 10 mars 1873 que le Conseil communal de Nyon nomme une commission de l'infirmerie.

Le 9 février 1874, le Conseil communal vote un crédit pour la fondation de l'infirmerie et offre, pour la faire, le bâtiment de l'hôpital pour 30 ans à la commission de l'infirmerie.

Ainsi l'hôpital qui avait commencé son activité charitable au début du $\mathrm{XIV}^{\mathrm{e}}$ siècle, se transformait en hôpital moderne à la fin du $\mathrm{XIX}^{\mathrm{e}}$ siècle.

Grâce à l'amabilité de Mr P.L. BADER, j'ai pu consulter les papiers privés, une sorte de journal, de Madame L. Bader, née Chevrier en 1859.

J'en ai tiré des remarques pleines de saveur à propos de l'hôpital.

«On traversait la cour pavée, mal entretenue. Dans une grande salle du rez-de-chaussée, il y avait quelques grands lits de bois à rideaux de coton blanc. Des hardes suspendues aux parois, quelques vaisselles sur des chaises dépaillées. Des toiles d'araignées partout.

«Pas de local spécial affecté à la passade qui abritait les Handwerksbursch, les Musiques de mineurs etc. Dans l'hiver 1870/71 les Bourbakis furent en assez grand nombre. Les internés malades étaient soignés pour la plupart à l'église sur la paille. Il ne me souvient pas si l'hôpital fut utilisé en cette circonstance. «Ceci nous montre bien quelle était la destination habi» tuelle de l'hôpital. Et maintenant nous trouvons des renseignements sur » la médication utilisée dans certains cas.» A cette époque et durant bien des années encore, ma grand-mère, Madame Chevrier-Scherer, confectionnait chaque printemps le fameux onguent Scherer qui guérissait infailliblement, les panaris, tours d'ongle, mals blancs de toute sorte à condition qu'on s'y prît à temps. Il y en avait un dépôt à l'hôpital.

«La poudre contre l'épilepsie et autres maladies nerveuses préparée par le Dr Chapuis, pharmacien, composée de taupes calcinées, était aussi largement répandue et distribuée par ma grand-mère à tous les malades des environs. Les femmes devaient apporter leur dé à coudre pour mesurer la dose.

«Nous apprenons aussi qu'il y eut à Nyon une épidémie de petite vérole en 1859 et qu'on la soignait en brûlant du vinaigre dans l'escalier. Il y eut aussi une épidémie de choléra en 1893; mais elle passa vite et ne fit pas beaucoup de victimes.» 
Il va sans dire qu'une telle longévité de l'établissement n'a pas été sans de nombreux événements dramatiques ou comiques; pour ne pas allonger cette communication, je n'en citerai que deux, qui, avec le recul du temps sont assez amusants à considérer. Le premier concerne les rapports fugitifs de l'hôpital avec l'Ordre de Malte.

Il y avait à l'église de Saint-Jean hors les murs une copie sommaire des Indulgences de l'église de Saint-Jean-de-Jérusalem, ensuite de Rhodes, ensuite de Malte. Cet ordre souverain et militaire était très puissant à l'époque. La connaissance de ce texte fit l'impression la plus forte sur les esprits de ces messieurs de la bourgeoisie de Nyon. Mais il semble bien que ce fut plus par vanité que par vraie piété qu'ils furent touchés. Tant et si bien qu'il fut décidé de s'adresser à la Commanderie de l'Ordre. "Ainsi le 17 avril 1491, une supplication dressée en Conseil Général assemblé dans l'église de SaintJean de Nyon, par son de cloche, est adressée aux Chevaliers de l'Ordre de Saint-Jean-de-Jérusalem, transférés alors en l'isle de Rhodes. Cette assemblée demande que l'hôpital soit honoré et doté des privilèges et honneurs conférés audit Ordre de Rhodes; que le recteur dudit hôpital de Nyon fut tenu porter l'habit dudit Ordre, que ledit hôpital jouît des privilèges et indulgences attachés aux églises dudit Ordre. Et enfin que ledit hôpital fut reçu et constitué sous la préceptorie de la Chaux, diocèse de Lausanne; en sorte que le recteur qui serait nommable à l'avenir, serait institué par ledit commandeur de la Chaux et serait résidence perpétuelle audit hôpital.»

Je n'ai pu trouver aux archives de Nyon le motif de la fin de non-recevoir qui fut réservé à cette supplication, car il n'est plus du tout question de l'affaire. Pour en avoir le cœur net, je me suis adressé à la Chancellerie de l'Ordre de Malte à Rome, il y a quelque temps. Mais je n'ai pas encore reçu de réponse à ma «supplication». Il est possible que les archives de l'Ordre ne possèdent pas non plus de document à ce sujet? Nous verrons.

Le second événement assez curieux, se place à peu près au même moment, puisqu'il date de 1497. La proximité dans le temps de ces deux événements me fait penser que l'ardeur des Nyonnais à augmenter leur capital d'indulgences, n'était pas absolument pur de toute autre préoccupation plus terre à terre, surtout en ce qui concerne le recteur de l'hôpital. Il semble bien que sous le couvert de grands avantages spirituels, se cachait un désir moins évangélique de biens matériels. Le fait de faire partie de l'Ordre de Malte, devait amener des fonds substantiels, qui devaient faire singulièrement défaut à ce moment là. 
La tentative envers l'Ordre de Malte ayant échoué, il fallait trouver autre chose; une manœuvre plus subtile fut engagée qui avait pour but de pouvoir bénéficier des ressources, non négligeables des confréries établies à Nyon. Voici comment l'affaire se passa et comment elle faillit réussir, pour le plus grand dam de trois confréries laïques nyonnaises. L'imagination du recteur n'était jamais mise en défaut.

Par un acte daté du 20 août 14.98 (acte non signé), on peut constater que les revenus de l'hôpital ne se montent plus qu'à dix florins. Mais on apprend en même temps que les revenus des Confréries de la Sainte-Trinité, de l'Eucharistie et du Saint-Esprit se montent à 20 florins. Il serait donc profitable de pouvoir unir ces confréries à l'hôpital pour en augmenter le revenu et par ce moyen subvenir aux pauvres hospitalisés.

Pour obtenir cette fusion, le recteur n'hésitera pas à user de moyens fort discutables. Avec le concours de Noble Jean de Usier, laïc, il réussit à obtenir du pape la consécration officielle de cette fusion.

« Jean de Usier prétendait être le seul membre restant de ces confréries, et il consentait à ce que lesdites trois confréries avec tous leurs droits et revenus fussent unies et annexées à l'hôpital, réservé le droit de patronat et de présentation du recteur, et que lesdits recteurs ne puissent rien aliéner. Ledit Noble Jean de Usier supplie le Saint-Siège d'admettre ladite union et incorporation. Et pour mener l'affaire à bien, il avait constitué à Rome trois procureurs pour suivre l'affaire.»

Il réussit.

Et les nyonnais avaient été doublement dupés, car la manœuvre avait commencé bien avant qu'ils en aient eu connaissance. En effet l'acte précédent est de 1498, mais la bulle du pape, lorsqu'elle arriva à Nyon était datée de 1497, le 10 mars; c'était l'an sixième du pontificat du Souverain Pontife, lequel était alors Alexandre VI, Borgia, dont on sait que le scrupule n'était pas non plus la vertu dominante (Alexandre VI pape de 1492 à 1503). Cette bulle apprenait aux Nyonnais qu'à l'instance de Vénérable Pierre Reverdil Recteur de l'hôpital de la Bien-Heureuse Vierge Marie de la ville de Nyon, le Saint-Siège avait décidé d'unir et incorporer les confréries de la Sainte-Trinité, de l'Eucharistie du Christ et du Saint-Esprit avec tous les droits et revenus à l'hôpital.

Mais chose encore plus curieuse, on y apprenait en même temps, que le Saint Siège n'avait pas jugé indispensable d'entendre les parties intéressées.

La ville de Nyon fit appel en cour de Rome en avançant les arguments suivants. (Le 19 janvier 1499.) 
$1^{\circ}$ - La ville de Nyon n'a pas été consultée et n'a jamais consenti à cette union.

$2^{\circ}$ - Vénérable Pierre Reverdil a faussement exposé le problème dans sa supplication.

$3^{\circ}-$ De plus il avait de quoi vivre honnêtement lui et l'hôpital, des revenus de ce dernier.

$4^{\circ}$ - Les confréries spoliées avaient été fondées et dotées par les bourgeois et les habitants de Nyon et par les autres paroissiens et aucun d'eux n'avait été consulté.

$5^{\circ}$ - Ces bourgeois et habitants s'étaient engagés à soutenir et maintenir l'hôpital.

$6^{\circ}$ - Quant à Jean de Usier, il n'était pas prieur des confréries, puisqu'il n'était même pas membre et encore bien moins l'unique et dernier membre.

$7^{\circ}$ - De par le règlement des confréries, une telle union ne pouvait se faire que du consentement de toutes les personnes intéressées.

En conclusion, la ville de Nyon demandait au Saint-Siège la révocation de la bulle du 10 mars 1497.

Elle finit par avoir gain de cause, mais l'alerte avait été chaude.

C'est la somme de toutes ces vanités comme de tous ces dévouements, la suite de ces longues années de malheur et de bonheur, de liberté et de servitude qui ont amené insensiblement les «hospitia» du Moyen-Age à nos hôpitaux modernes.

J'espère avoir, dans une modeste mesure, indiqué ce tortueux et long cheminement et contribué ainsi à donner un peu plus de lumière à ces siècles oubliés. 